\title{
Application and Mechanism of Ferrihydrite in the EDDS Improved Heterogeneous Photo-Fenton System: the Role of Different Reactive Species Under Different Conditions
}

\author{
Hongjie Xie ${ }^{a}$, Mengqi Luo ${ }^{a}$, Wenyu Huang ${ }^{a, d} *$, Ying Huang ${ }^{a}, X^{2}$ iaoqing Feng ${ }^{a}$, Zisong Xu ${ }^{a}$, Wei Luo ${ }^{b}$, \\ Shuangfei Wang ${ }^{c, d}$, Hongfei Lin ${ }^{d}$, Gilles Mailhot ${ }^{e}$
}

\begin{abstract}
The application of carboxylic acid and natural iron oxide in heterogeneous AOP systems for the treatment of organic pollutants in water has attracted extensive attention. In this work, ethylenediamine-N,N'-disuccinic acid (EDDS) was complexed with ferrihydrite to improve the photo-Fenton system for bisphenol A (BPA) degradation, and the mechanism of the system was investigated. TEM, XRD and BET were used to investigate the morphology, structure and specific surface area of ferrihydrite. The characterization results revealed that ferrihydrite was amorphous and agglomerated, and its main composition was 2-2lfh, and its specific surface area was $297.5 \mathrm{~m}^{2} \mathrm{~g}^{-1}$. BPA degradation experiments, carried out at different influence factors including the initial concentration of EDDS, amount of ferrihydrite and initial concentration of $\mathrm{H}_{2} \mathrm{O}_{2}$, showed a maximum degradation rate of BPA of $85.1 \%$ under the optimal conditions (at $1.0 \mathrm{mmol} \mathrm{L}^{-1}$ of EDDS, $0.6 \mathrm{~g} \mathrm{~L}^{-1}$ of ferrihydrite and $0.5 \mathrm{mmol} \mathrm{L}^{-1}$ of $\mathrm{H}_{2} \mathrm{O}_{2}$ ). Finally, in order to analyze the mechanism of BPA degradation, 2-propanol and chloroform were used as free radical scavengers to capture hydroxyl radicals $(\cdot \mathrm{OH})$ and superoxide anion radicals $\left(\cdot \mathrm{O}_{2}{ }^{2}\right)$ respectively. The results indicated that $\cdot \mathrm{OH}$ radicals and ${ }^{1} \mathrm{O}_{2}$ play an important role in aerobic conditions, while $\cdot \mathrm{O}_{2}{ }^{-}$radicals produced by the reaction of $\mathrm{H}_{2} \mathrm{O}_{2}$ and holes are non-negligible in anaerobic conditions. Moreover, the iron dissolution also plays a promoting role in BPA degradation. Overall, $\mathrm{Fe}$ (III) and Fe( II) redox processes are accelerated due to the $\mathrm{Fe}$ (III)EDDS complex reaction under irradiation and the presence of $\mathrm{O}_{2}$ radicals and trace iron dissolution, which increases the number of reactive species in the system. The BPA degradation is facilitated by various reactive species including $\cdot \mathrm{OH}$ radicals, ${ }^{1} \mathrm{O}_{2}$ and $\cdot \mathrm{O}_{2}{ }^{-}$radicals.
\end{abstract}

\section{Introduction}

Advanced Oxidation Processes (AOPs) have been proposed as promising tertiary wastewater treatments for micropollutant removal. Among AOPs, the photo-Fenton process has been confirmed to increase the Fenton reaction rate through the introduction of UV or visible light.

In the Fenton or photo-Fenton process, iron oxide has been widely used because of its excellent catalytic performance, thereby overcoming the drawback of the traditional Fenton process. In the traditional Fenton reaction, the most suitable $\mathrm{pH}$ value would be $<3$ in order to maintain the system stability and promote the production of $\cdot \mathrm{OH}$ radicals production. Furthermore, the conventional homogeneous photo-Fenton reactions have very low oxidation performance because of the low rate of $\mathrm{Fe}^{2+}$ regeneration. ${ }^{1}$ In recent studies, a large number of iron oxide species have been considered, such as $\mathrm{Fe}_{3} \mathrm{O}_{4},{ }^{2} \alpha-$ $\mathrm{Fe}_{2} \mathrm{O}_{3},{ }^{3} \alpha-\mathrm{FeOOH},{ }^{4} \beta-\mathrm{FeOO}_{5}$ and $\gamma-\mathrm{Fe}_{2} \mathrm{O}_{3}{ }^{6}$. Among them, ferrihydrite has attracted significant research interest. ${ }^{7-9}$ Ferrihydrite is widely distributed in the biosphere, and it is also a precursor of other crystalline iron oxides in the environment. Additionally, ferrihydrite has a large specific surface area and high surface activity. ${ }^{7}$ While the exact chemical composition of ferrihydrite is not currently confirmed, it is proposed to be $\mathrm{Fe}_{5} \mathrm{HO}_{8} \cdot 4 \mathrm{H}_{2} \mathrm{O}{ }^{8}$ In order to improve the system, many studies have focused on introducing different iron oxides including ferrihydrite into the photo-Fenton system to facilitate the conversion rate between $\mathrm{Fe}^{3+}$ and $\mathrm{Fe}^{2+}$. These include

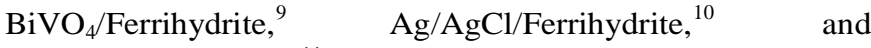
$\mathrm{Ag} / \mathrm{AgBr} / \mathrm{Ferrihydrite}^{11}$. It has been proven that ferrihydrite can catalyze $\mathrm{H}_{2} \mathrm{O}_{2}$ to produce $\cdot \mathrm{OH}$ radicals to act on pollutants.

Organic chelating agents (CAs), such as ethylene diaminetetraacetic acid (EDTA), nitrilotriacetic acid (NTA), 1,10-Phenanthroline hydrate, ammonium citrate, sodium potassium tartrate, and dithizone are usually used to remove metal impurities in water. ${ }^{12-15}$ Recent studies have shown that CAs can be used in the photo-Fenton process to promote the degradation efficiency, especially at a neutral $\mathrm{pH} .{ }^{16}$ Among them, aminocarboxylic acids such as EDTA, DTPA were first

\footnotetext{
a. School of Resources, Environment and Materials, Guangxi University, 100 Daxue East Road, Nanning 530004, China

b. Department of Municipal Engineering, Southeast University, Nanjing 211189 China

c. Guangxi Key Laboratory of Clean Pulp \& Papermaking and Pollution Control, Guangxi University, Nanning 530004, China

d. Guangxi Bossco Environmental Protection Technology Co.,Ltd, Nanning, 530007, China

e. Université Clermont Auvergne, CNRS, SIGMA Clermont, Institut de Chimie de Clermont-Ferrand, F-63000 Clermont-Ferrand, France

* Corresponding Author:Wenyu Huang, Ph.D

School of Resources, Environment and Materials of Guangxi University, Nanning,
} 530004,P.R.China. Email: huangwenyu@gxu.edu.cn 
used, however, they were found to be biotoxic and nonbiodegradable. ${ }^{17}$ Therefore, there is a research opportunity to find chelating agents that can be biodegradable and nontoxic.

Ethylenediamine-N, $\mathrm{N}^{\prime}$-disuccinic acid (EDDS) is one of CAs, which is environmentally friendly and non-toxic to the environment and biological systems. Furthermore, EDDS is considered a substitute for harmful chelates. In previous studies, EDDS was used to complex Fe(III) to form a stable combination. ${ }^{18}$ The complex system of Fe(III)-EDDS was applied in the photo-Fenton process to improved oxidation performance in a broad $\mathrm{pH}$ range.

Bisphenol A (BPA) is a typical environmental endocrine disruptor, which disrupts the mechanism of endogenous hormones in human and animal bodies, thus affecting the normal growth and reproduction of organisms. ${ }^{19-21}$ BPA is used in plastics, medical devices, automobile production and so on, it is thus widely used in industries and detectable in natural environment. At present, the degradation methods of BPA mainly include biological treatment, physical treatment and chemical treatment. Among the chemical treatment methods, AOPs have proven to be particularly efficient for BPA degradation.

For these reasons, the degradation performance of BPA in the EDDS-ferrihydrite photo-Fenton system is studied in this research. The morphology and structure of ferrihydrite were characterized using different methods and used to analyze the system mechanism. The effects of different parameters on BPA degradation such as initial concentration of EDDS, ferrihydrite amount and the initial concentration of $\mathrm{H}_{2} \mathrm{O}_{2}$ were studied. In order to further understand the degradation mechanism, the contribution of free radicals such as $\cdot \mathrm{OH}$ and $\cdot \mathrm{O}_{2}{ }^{-}$radicals were investigated, and the effect of iron dissolution on the oxidation process was assessed. The difference in the mechanism between in the aerobic and anaerobic conditions was determined based on the effect of different radicals.

\section{Experimental}

\section{Chemicals}

Sodium hydroxide, hydrochloric acid, potassium hydroxide, ferric nitrate nonahydrate, hydrogen peroxide (30wt\%) were obtained from the Chengdu Kelong Chemical Reagent Factory. BPA was from the Shanghai Ziyi Reagent Factory. Isopropanol and chloroform were from the Guangxi kezan biological co. LTD. S, $\mathrm{S}^{\prime}$-ethylenediamine-N, $\mathrm{N}^{\prime}$-disuccinic acid trisodium salt (EDDS) was obtained from Sigma. Milli-Q water was used for all experiments.

\section{Synthesis and characterization of ferrihydrite}

Ferrihydrite was synthesized as follows ${ }^{22}$ : $40.0 \mathrm{~g}$ $\mathrm{Fe}\left(\mathrm{NO}_{3}\right)_{3} \cdot 9 \mathrm{H}_{2} \mathrm{O}$ was dissolved in $500 \mathrm{~mL}$ of Milli-Q water, and $310 \mathrm{~mL} \mathrm{KOH}\left(1 \mathrm{~mol} \mathrm{~L}^{-1}\right)$ was added at the rate of $100 \mathrm{~mL} \mathrm{~min}^{-1}$. The $\mathrm{pH}$ value was adjusted using $1 \mathrm{~mol} \mathrm{~L}^{-1} \mathrm{KOH}$ to approximately 7.5. The system was then stirred violently for 1 hour. The obtained colloids were centrifuged for 12 minutes
(4000 RPM) and the supernatant was discarded. The solid was washed with Milli-Q water and then centrifuged for 12 minutes. The procedure was repeated 5 times, and finally the solid was dried at approximately $40^{\circ} \mathrm{C}$.

The morphology of ferrihydrite was observed by transmission electron microscopy (TEM) using a JEM-2100F TEM (JEOL, Japan). Before the test, the ferrihydrite was put into a small beaker with ethanol. The sample was then suspended and dispersed by ultrasonic oscillation. After that, a drop of suspension was dropped onto the carbon-support copper-film network, and the test was conducted once after the ethanol had evaporated.

The crystal structure of ferrihydrite was analyzed by X-ray powder diffraction (XRD), and the XRD model is DX-2700A (HAOYUAN, China). The measurement conditions are as follows: XRD was performed at $40 \mathrm{KV}$ voltage and $100 \mathrm{~mA}$ current with $2 \theta / \theta$ coupling continuous scanning with a step width of 0.02 , and a scanning speed of $10^{\circ} \mathrm{min}^{-1}$.

The specific surface area of ferrihydrite was analyzed by Brunauer Emmett Teller (BET) analysis using a SSA-3600 (BEODO, Beijing). Before analysis, an appropriate amount of ferrihydrite was placed in a special glass tube and then analyzed in the SSA-3600 automatic specific surface and pore analyzer.

\section{Photo-Fenton experiments}

The experiments were carried out in a home-made photoreactive apparatus consisting of a cylindrical container made of acrylic materials. The experimental lamp was a HPI-T Plus 250W metal halide lamp with a continuous emission spectrum from $290-800 \mathrm{~nm}$, which was used to simulate solar illumination. The temperature and stability of the lamp was controlled using circulating water. An aeration unit was introduced at the bottom of the reaction container to give a fully mixed solution and to provide oxygen.

Before reaction, BPA and EDDS were added to the reaction solution and the $\mathrm{pH}$ was adjusted. The prepared reaction solution and ferrihydrite were added to the photoreactive apparatus, and then a certain amount of $\mathrm{H}_{2} \mathrm{O}_{2}$ was added to the reactor. Timing started after turning on the light and aeration. Samples were taken for centrifugation at intervals, and the supernatant was filtered with a $0.45 \mathrm{pm}$ filter membrane and placed in the liquid chromatograph for detection.

To investigate the role of free radicals and the mechanism of degradation in the experiment, a radical scavenger was used to capture the free radicals produced 2-propanol and chloroform were used as $\cdot \mathrm{OH}$ and $\cdot \mathrm{O}_{2}{ }^{-}$scavengers, respectively. ${ }^{23} \mathrm{CF}$ is highly reactive with $\cdot \mathrm{O}_{2}^{-} \quad\left(\mathrm{k}_{\cdot \mathrm{O} 2}{ }_{\mathrm{C}}^{-} \mathrm{CF}=3.0 \times 10^{10} \mathrm{~mol}^{-1} \mathrm{Ls}^{-1}\right)$ and poorly reactive with $\cdot \mathrm{OH}\left(\mathrm{k} \cdot \mathrm{OH}, \mathrm{CF}=5.0 \times 10^{6} \mathrm{~mol}^{-1} \mathrm{Ls}^{-1}\right){ }^{24}$

\section{Analytical methods}

The concentration of BPA was determined by UHPLC-CLASS (WATERS, USA) ultra-high-performance liquid chromatography. The experiments were performed by UV detection at $275 \mathrm{~nm}$ with a under the column temperature of $40^{\circ} \mathrm{C}$. The retention time of BPA was 3 minutes. The flow rate 
was $0.2 \mathrm{~mL} \mathrm{~min}^{-1}$ and the mobile phase was a mixture of methanol and water (65:35).

The degradation rate of BPA was calculated using the following formula :

$$
\left(1-\mathrm{C}_{\mathrm{t}} / \mathrm{C}_{0}\right) \times 100 \% \text {, }
$$

where $C_{t}$ refers to the concentration of BPA at some point during degradation, and $\mathrm{C}_{0}$ refers to the initial concentration of BPA.

The content of ferrous iron $\left(\mathrm{Fe}^{2+}\right)$ and total iron was determined by the o-phenantholine method. ${ }^{25}$ The principle of determination is that $\mathrm{Fe}^{2+}$ forms a stable orange-red complex with o-phenanthroline in solution at a $\mathrm{pH}$ between 3 and 9. The total iron required was determined by adding $1 \mathrm{~mL}$ hydroxylamine hydrochloride to reduce the ferric ion $\left(\mathrm{Fe}^{3+}\right)$ to $\mathrm{Fe}^{2+}$.

\section{Results and discussion}

\section{Characterization of ferrihydrite}

The morphologies of ferrihydrite were investigated by TEM (Fig. 1). The agglomeration phenomenon of the ferrihydrite particles failed to reveal the shapes and morphology of single particles and individual crystals. Ferrihydrite is an amorphous granular polymer and is extremely fine grained, thus it is difficult to distinguish the morphology of individual particles. ${ }^{26-}$ ${ }^{28}$ The XRD patterns of ferrihydrite were examined (Fig. 2). As shown in Fig. 2, there are two weak, broad peaks at $2 \theta=$ $34.65^{\circ}, 61.24^{\circ}$. These results are consistent with the diffraction peak of 2-line ferrihydrite (2-lfh) which is regarded as the typical species of ferrihydrite, ${ }^{29,30}$ indicating that the main component of the sample is 2-lfh. Compared with other ferrihydrite species, such as 6-lfh and 3-lfh, 2-lfh has weak crystallinity and a specific surface area, which increases the number of reactive sites and the catalytic activity toward $\mathrm{H}_{2} \mathrm{O}_{2}{ }^{31}$ As determined by BET, the specific surface area of the synthesized ferrihydrite in this study was $297.5 \mathrm{~m}^{2} \mathrm{~g}^{-1}$, which is larger than those obtained in previous studies. ${ }^{32,33}$ The high specific surface area means more reactive sites available hydrogen peroxide catalysis. Therefore, this characteristic is beneficial for increasing the reaction rate of the photo-Fenton process.

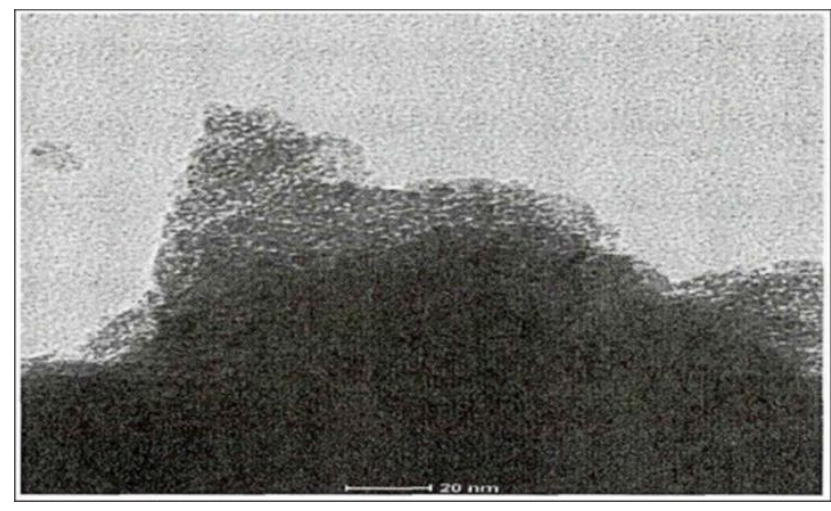

Fig. 1 TEM image of ferrihydrite

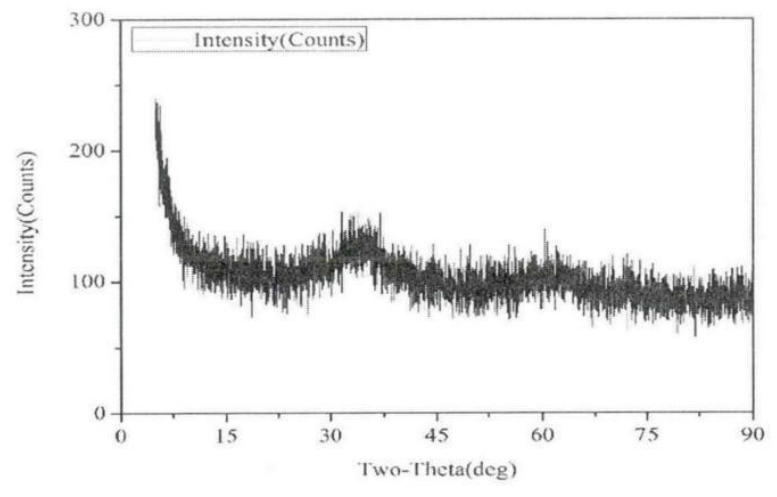

Fig. 2 XRD diffraction pattern of ferrihydrite

\section{Effects of different factors on BPA degradation}

Effects of EDDS Concentration. As shown in Fig. 3a, the effect of EDDS concentration on BPA degradation in the modified photo-Fenton system was discussed. The degradation rate of BPA increased from $81.6 \%$ to $85.1 \%$ when the concentration of EDDS increased from 0.5 to $1.0 \mathrm{mmol} \mathrm{L}^{-1}$. However, the degradation rate of BPA decreased at higher concentrations of EDDS ( 1.5 to $2.5 \mathrm{mmol} \mathrm{L}^{-1}$ ) which could be caused by two possible reasons. On the one hand, the ligand metal charge transfer reduced $\mathrm{Fe}(\mathrm{III})$ to $\mathrm{Fe}(\mathrm{II})$. Therefore, with the increase of the initial EDDS concentration to $1.0 \mathrm{mmol} \mathrm{L}^{-1}$, the circulation between $\mathrm{Fe}(\mathrm{III})-\mathrm{Fe}(\mathrm{II})$ is promoted (see Eqs. (2)(5))..$^{34,35}$ On the other hand, with the increase of EDDS concentration, the $\mathrm{Fe}$ (III)-EDDS complex could promote the photochemical reaction rapidly and produce reactive radicals such as $\cdot \mathrm{OH}$ and $\cdot \mathrm{O}_{2}{ }^{-}$in the presence of UV radiation. ${ }^{18,36,37}$

$$
\begin{aligned}
& \mathrm{Fe}(\text { III)+EDDS } \rightarrow \mathrm{Fe} \text { (III)-EDDS } \\
& \mathrm{Fe} \text { (III)-EDDS }+\mathrm{hv} \rightarrow \mathrm{Fe} \text { ( II )-EDDS } \\
& \mathrm{Fe}(\text { II }) \text {-EDDS } \cdot \rightarrow \mathrm{Fe} \text { ( II )+EDDS } \\
& \mathrm{Fe}(\mathrm{II})+\mathrm{H}_{2} \mathrm{O}_{2} \rightarrow \mathrm{Fe}(\mathrm{III})+\cdot \mathrm{OH}+\mathrm{OH}^{-} \\
& \mathrm{EDDS}+\cdot \mathrm{OH} \rightarrow \text { photoproducts }
\end{aligned}
$$

However, if the EDDS concentration is too high, it inhibits the degradation of BPA. This may be due to the competitive reaction between EDDS and BPA on $\cdot \mathrm{OH}\left(\mathrm{k}_{\mathrm{EDDS}} \cdot \mathrm{OH}=2.0 \times 10^{8}\right.$ $\mathrm{M}^{-1} \mathrm{~S}^{-1}$ ), according to reaction (6). ${ }^{38}$

Effects of the ferrihydrite loading. Fig. $3 b$ shows the impact of different ferrihydrite loadings on BPA degradation. The degradation rate of BPA increased when ferrihydrite dosage increased up to $0.6 \mathrm{~g} \mathrm{~L}^{-1}$, and then decreased with further dosage increase. With a low ferrihydrite dose, the active sites on the surface of ferrihydrite are saturated quickly, and thus the interaction between ferrihydrite and other reactants is limited, reducing the BPA degradation. With the increase of ferrihydrite dosage, the increase of surface active sites increased the generation of $\cdot \mathrm{OH}$, which enhanced the degradation efficiency of BPA. ${ }^{39}$ Moreover, the increase of ferrihydrite dose improved the utilization of irradiation and increased dissolved iron content. ${ }^{40,41}$ However, the decrease in the degradation rate of BPA at the highest ferrihydrite doses may be due to the rapid degradation of EDDS when the Fe(III)-EDDS complex was formed. ${ }^{18}$ Therefore, the Fe(III)-EDDS complex would not be 
formed in solution, resulting in low BPA degradation rates Another possibility is that $\cdot \mathrm{OH}$ radicals could be scavenged by the ferrihydrite surface. Iron oxide surfaces can react with $\cdot \mathrm{OH}$ radicals $\left(\mathrm{k}=8 \times 10^{11}\left(\mathrm{~g} \cdot \mathrm{mL}^{-1}\right)^{-1} \mathrm{~s}^{-1}\right) \quad$ according to previous research. $^{42}$

Effects of $\mathrm{H}_{2} \mathrm{O}_{2}$ Concentration. Fig. 3c reveals the effect of different $\mathrm{H}_{2} \mathrm{O}_{2}$ concentrations on BPA degradation. The increased of $\mathrm{H}_{2} \mathrm{O}_{2}$ concentration firstly led to a slight increase in BPA degradation, while additional $\mathrm{H}_{2} \mathrm{O}_{2}$ reduced the rate. This result could be due to the scavenging effect of $\mathrm{H}_{2} \mathrm{O}_{2}$ on . $\mathrm{OH}$ radicals. In the low $\mathrm{H}_{2} \mathrm{O}_{2}$ concentration range, the increase of $\mathrm{H}_{2} \mathrm{O}_{2}$ concentration augmented the production of $\cdot \mathrm{OH}$ radicals, thereby improving the degradation efficiency of BPA. . OH radicals were produced in two different pathways. Pathway one is the photolysis of $\mathrm{H}_{2} \mathrm{O}_{2}$ to produce $\cdot \mathrm{OH}$ radicals, and pathway two is the Fenton process (see Eqs. (7), (5)). ${ }^{43}$

$$
\begin{gathered}
\mathrm{H}_{2} \mathrm{O}_{2}+\mathrm{hv} \rightarrow 2 \cdot \mathrm{OH} \\
\cdot \mathrm{OH}+\mathrm{H}_{2} \mathrm{O}_{2} \rightarrow \mathrm{HO}_{2} \cdot+\mathrm{H}_{2} \mathrm{O} \\
\mathrm{HO}_{2} \cdot+\cdot \mathrm{OH} \rightarrow \mathrm{H}_{2} \mathrm{O}+\mathrm{O}_{2}
\end{gathered}
$$

However, excessive $\mathrm{H}_{2} \mathrm{O}_{2}$ will react with $\cdot \mathrm{OH}$ radicals to form $\cdot \mathrm{O}_{2}^{-}$radicals with weaker oxidation ability, which cannot further oxidize BPA and its degradation intermediates (see Eqs. (8)-(9) ${ }^{44}$ In fact, Li et al. ${ }^{45}$ also got a similar conclusion that . $\mathrm{OH}$ radicals could be scavenged by excessive $\mathrm{H}_{2} \mathrm{O}_{2}$ in the nano- $\mathrm{Fe}_{3} \mathrm{O}_{4} / \mathrm{UV}-$ Fenton system. As a result, the degradation rate of BPA gradually increased below the optimal concentration and decreased above this concentration.
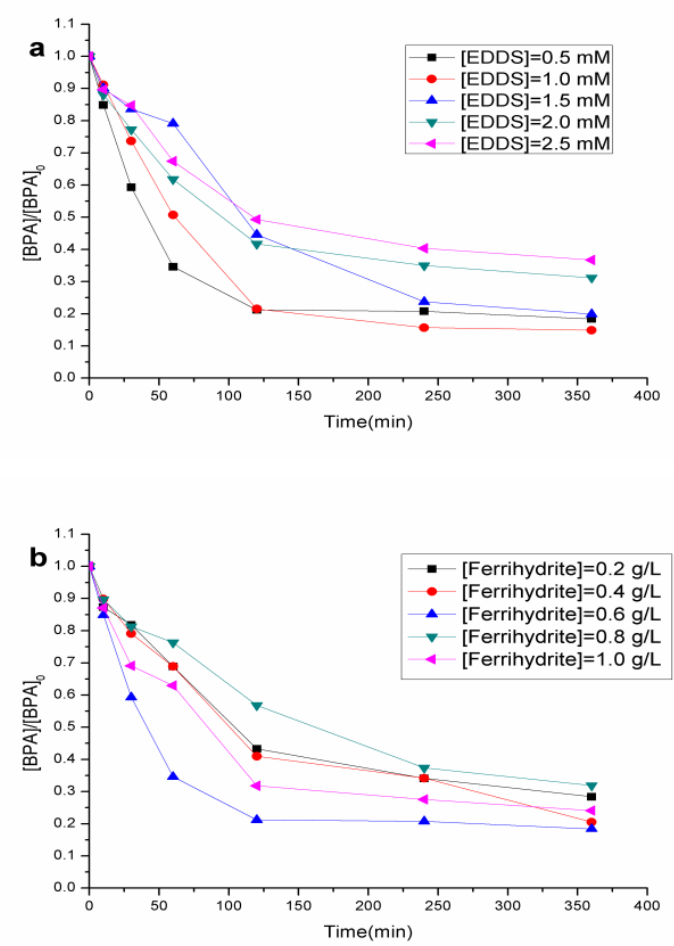

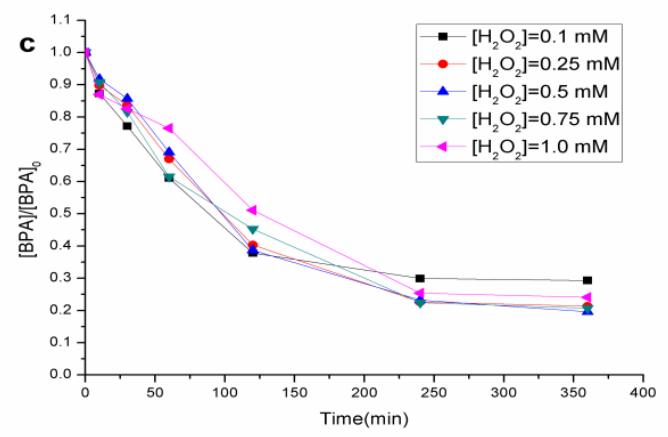

Fig. 3 Effects of BPA degradation in the modified photoFenton systems at a EDDS concentration (conditions: [ferrihydrite] $=0.6 \mathrm{~g} \mathrm{~L}^{-1},\left[\mathrm{H}_{2} \mathrm{O}_{2}\right]=0.5 \mathrm{mmol} \mathrm{L}^{-1},[\mathrm{BPA}]_{0}=20 \mu \mathrm{mol} \mathrm{L}$ ${ }^{1}, \mathrm{pH}=7$ ), $\mathbf{b}$ ferrihydrite loading (conditions: [EDDS] $=1.0 \mathrm{mmol} \mathrm{L}^{-}$ ${ }^{1},\left[\mathrm{H}_{2} \mathrm{O}_{2}\right]=0.5 \mathrm{mmol} \mathrm{L}^{-1},[B P A]_{0}=20 \mu \mathrm{mol} \mathrm{L}^{-1}, \mathrm{pH}=7$ ), and $\mathrm{c} \mathrm{H}_{2} \mathrm{O}_{2}$ concentration ([EDDS] $=1.0 \mathrm{mmol} \mathrm{L}^{-1}$, [ferrihydrite] $=0.6 \mathrm{~g} \mathrm{~L}^{-1}$, $\left.[B P A]_{0}=20 \mu \mathrm{mol} \mathrm{L}^{-1}, \mathrm{pH}=7\right)$.

\section{Mechanism analysis of BPA degradation}

BPA degradation in different systems. In order to understand the role each factor plays, including irradiation and EDDS, BPA degradation was carried out in different systems with the same conditions, and these results are shown in Fig. 4. Obviously, these results suggest that irradiation contributes significantly to the BPA oxidation process. All the systems with irradiation yielded more efficient BPA degradation than those without irradiation. This enhancement could be attributed to the reduction of $\mathrm{Fe}$ (III) to $\mathrm{Fe}$ (II) induced by irradiation, which increases the circulation between $\mathrm{Fe}$ (III) and Fe(II), ${ }^{46}$ and then improves the generation of $\cdot \mathrm{OH}$. In addition, the photoactivity of ferrihydrite could enhance the interaction between its surface-active sites and $\mathrm{H}_{2} \mathrm{O}_{2}$ and also increase the production of $\cdot \mathrm{OH}$ under irradiation. Secondly, it is possible that complexation between EDDS and ferrihydrite occurred and hence the photoactivity was promoted. ${ }^{18,47}$ Moreover, the BPA degradation efficiency with aeration was higher than without aeration. This is likely due to the presence of singlet oxygen $\left({ }^{1} \mathrm{O}_{2}\right)$ molecules which also contribute to BPA degradation. According to literature ${ }^{48}$ iron oxides can generate electron-hole pairs in the presence of irradiation and oxygen, and generate ${ }^{1} \mathrm{O}_{2}$ indirectly due to the strong oxidation of the holes. Based on the reaction rate constant between $\mathrm{BPA}$ and ${ }^{1} \mathrm{O}_{2} \quad\left(\mathrm{k}_{\mathrm{BPA}}\right.$, $\left.{ }_{102}=(2.15 \pm 0.017) \times 10^{8} \mathrm{M}^{-1} \mathrm{~s}^{-1}\right),{ }^{49} \mathrm{BPA}$ can be directly oxidized by ${ }^{1} \mathrm{O}_{2}$ to $\mathrm{H}_{2} \mathrm{O}$ and $\mathrm{CO}_{2}$. 


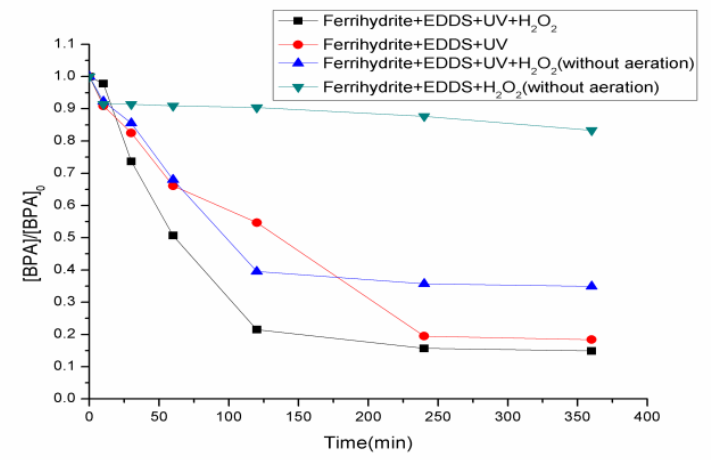

Fig. 4 BPA degradation in different systems (conditions: [EDDS] $=1.0 \mathrm{mmol} \mathrm{L}^{-1}$, [ferrihydrite] $=0.6 \mathrm{~g} \mathrm{~L}^{-1},\left[\mathrm{H}_{2} \mathrm{O}_{2}\right]=0.5 \mathrm{mmol}$ $\mathrm{L}^{-1},[\mathrm{BPA}]_{0}=20 \mu \mathrm{mol} \mathrm{L}^{-1}, \mathrm{pH}=7$ )

Contribution of different reactive species. To better understanding the role of different reactive species in the ferrihydrite-EDDS/photo-Fenton system, radicals scavenging experiments were carried out and the degradation of BPA was investigated. Firstly, 2-propanol was used as a $\cdot \mathrm{OH}$ radicals scavenger to determine the effects of. $\mathrm{OH}$ radicals. ${ }^{50-52}$ Different concentrations of 2-propanol were added to the solution. As showed in Fig. 5a, it is clear that the degradation efficiency of BPA decreased gradually with the increase of 2propanol concentration. When the 2-propanol concentration increased from $0 \mathrm{mmol} \mathrm{L}^{-1}$ to $10.0 \mathrm{mmol} \mathrm{L}^{-1}$ in the system, the BPA degradation percentage decreased from $85.1 \%$ to $40.0 \%$. This indicates that $\cdot \mathrm{OH}$ radicals play an important role in the oxidation processes. However, BPA degradation still occurred when $10.0 \mathrm{mmol} \mathrm{L}^{-1}$ of 2-propanol was used, a concentration where $\cdot \mathrm{OH}$ radicals should be completely captured by 2propanol theoretically $\quad\left(\mathrm{k}_{2-\mathrm{propanol}, \mathrm{HO}}=2 \times 10^{9} \mathrm{M}^{-1} \mathrm{~S}^{-1}\right.$, $\left.\mathrm{k}_{\mathrm{BPA}, \mathrm{HO}}=6.9 \times 10^{9} \mathrm{M}^{-1} \mathrm{~S}^{-1}\right),{ }^{53,54}$ indicating that other reactive species are responsible for direct BPA degradation directly in the system. 2-propanol with the same concentration of $10.0 \mathrm{mmol} \mathrm{L}^{-1}$ was used to remove $\cdot \mathrm{OH}$ radicals under the condition of magnetic stirring instead of aeration in order to distinguish the role of $\cdot \mathrm{OH}$ radicals and singlet oxygen $\left({ }^{1} \mathrm{O}_{2}\right)$. The results showed that the degradation percentage of BPA decreased to $17.9 \%$, and the inhibition effect was enhanced, suggesting that the role of ${ }^{1} \mathrm{O}_{2}$ in BPA degradation in this novel system could not be ignored. These results also demonstrated that ${ }^{1} \mathrm{O}_{2}$ can promote the degradation of BPA in Fe(III)-EDDS when applied in the reaction of homogeneous Fenton process. ${ }^{55}$ Torres ${ }^{56}$ examined the photodegradation mechanism of BPA and found that oxygen promoted the formation of BPA cationic free radicals $\left(\mathrm{BPA}^{+}\right)$, which accelerated the degradation of BPA(see Eqs. (10)-(12)). In addition, the presence of oxygen could enhance the formation of $\cdot \mathrm{OH}$ radicals via a series of further reactions, as shown in Eqs. (13)-(15). ${ }^{35,36}$

$\cdot \mathrm{OH}+\mathrm{BPA} \rightarrow \mathrm{BPA}-\mathrm{OH}$.

$\mathrm{BPA}-\mathrm{OH} \cdot+\mathrm{O}_{2} \rightarrow \mathrm{BPA}-\mathrm{OH}^{+}+{ }^{+} \cdot \mathrm{O}_{2}$

BPA-OH. ${ }^{+} \ldots \ldots \rightarrow \mathrm{CO}_{2}+\mathrm{H}_{2} \mathrm{O}$

EDDS $\cdot+\mathrm{O}_{2} \rightarrow$ product $+\cdot \mathrm{O}_{2}$

$\cdot \mathrm{O}_{2}{ }^{-}+\mathrm{HO}_{2} \cdot \mathrm{H}_{2} \mathrm{O} \rightarrow \mathrm{H}_{2} \mathrm{O}_{2}+\mathrm{O}_{2}+\mathrm{OH}^{-}$

$$
\mathrm{Fe} \text { (II ) }+\mathrm{H}_{2} \mathrm{O}_{2} \rightarrow \mathrm{Fe}(\text { III) }+\cdot \mathrm{OH}+\mathrm{OH}
$$

Furthermore, chloroform was applied to scavenge $\cdot \mathrm{O}_{2}^{-}$ radicals to understand the impact of $\cdot \mathrm{O}_{2}^{-}$radicals on BPA degradation. ${ }^{57}$ Different concentrations of chloroform were added to the solution as shown in Fig. 5b. When the chloroform concentration in the system, with air bubbling, was increased from 0 to $1.0 \mathrm{mmol} \mathrm{L}^{-1}$, the degradation rate of BPA increased from 85.1 to $86.2 \%$ after $6 \mathrm{~h}$, suggesting that the role of $\cdot \mathrm{O}_{2}$ radicals is negligible in BPA degradation in aerobic conditions. In contrast, the presence of $1.0 \mathrm{mmol} \mathrm{L}^{-1}$ chloroform under anaerobic conditions clearly inhibited BPA degradation, reducing it from $85.1 \%$ to $62.8 \%$, indicating the non-negligible role of $\cdot \mathrm{O}_{2}^{-}$radicals on BPA degradation under anaerobic conditions. In the Fenton mechanism, $\cdot \mathrm{O}_{2}^{-}$radicals are a strong reductants and promote the reduction of $\mathrm{Fe}$ (III) to $\mathrm{Fe}$ (II), which contributes to the generation of $\cdot \mathrm{OH}$ radicals (see Eqs. (16), (5)).$^{57}$ Therefore, the use of chloroform as the $\cdot \mathrm{O}_{2}{ }^{-}$scavenger may hinder the reaction between $\mathrm{Fe}(\mathrm{III})$ and $\cdot \mathrm{O}_{2}{ }^{-}$radicals (Eqs. (16)), which causes a reduction in $\mathrm{Fe}(\mathrm{II})$ and $\cdot \mathrm{OH}$ radicals production. Accordingly, there is a difference in the mechanism of BPA degradation under aerobic and anaerobic conditions, ${ }^{1} \mathrm{O}_{2}$ plays an important role in the former condition while $\cdot \mathrm{O}_{2}{ }^{-}$ radicals is non-negligible in the latter condition. In the aerobic condition, part of $\cdot \mathrm{O}_{2}^{-}$radicals are generated by the reduction reaction of oxygen and electron. ${ }^{58}$ Subsequently, $\cdot \mathrm{O}_{2}^{-}$radicals are immediately deactivated and converted to ${ }^{1} \mathrm{O}_{2}$ due to the oxidation of holes (see Eqs. (17)-(19)). ${ }^{48}$ Since $\mathrm{O}_{2}$ is usually the primary reducible compound in the system, the yield of $\cdot \mathrm{O}_{2}$ radicals is significantly higher in the primary steps of the photoFenton reaction, which further promotes a high ${ }^{1} \mathrm{O}_{2}$ production. ${ }^{59}$ In addition, a small amount of $\cdot \mathrm{O}_{2}{ }^{-}$radicals are generated in the system from $\mathrm{H}_{2} \mathrm{O}_{2}$ (see Eq. (20)), ${ }^{59}$ but with lower yields compared to reactions (18)-(19). Therefore, under aerobic conditions, high-yield ${ }^{1} \mathrm{O}_{2}$ plays a primary role in the degradation of BPA, while the role of $\cdot \mathrm{O}_{2}^{-}$radicals is negligible. However, the ${ }^{1} \mathrm{O}_{2}$ from (eqs (18)-(19)) is absent in the anaerobic conditions. Conversely, there are enough holes at the ferrihydrite surface to support the reaction of generating $\cdot \mathrm{O}_{2}{ }^{-}$radicals (see Eq. (20)). The formation of $\cdot \mathrm{O}_{2}{ }^{-}$radicals in large quantities promotes the redox reaction with $\mathrm{Fe}(\mathrm{III})$, and further facilitates the generation of $\cdot \mathrm{OH}$ radicals. Consequently, the $\cdot \mathrm{O}_{2}{ }^{-}$radicals are non-negligible in anaerobic conditions.

$$
\begin{aligned}
& \mathrm{Fe}(\text { III })+\cdot \mathrm{O}_{2}^{-} \rightarrow \mathrm{Fe}(\text { II })+\mathrm{O}_{2} \\
& \mathrm{Fe}(\mathrm{III})-\mathrm{EDDS}+\mathrm{hv} \rightarrow \mathrm{Fe}(\text { III })-\mathrm{EDDS}+\mathrm{e}^{-}+\mathrm{h}^{+} \\
& \mathrm{e}^{-}+\mathrm{O}_{2} \rightarrow \cdot \mathrm{O}_{2}^{-} \\
& \cdot \mathrm{O}_{2}^{-}+\mathrm{h}^{+} \rightarrow{ }^{1} \mathrm{O}_{2} \\
& \mathrm{H}_{2} \mathrm{O}_{2}+\mathrm{h}^{+} \rightarrow \cdot \mathrm{O}_{2}^{-}
\end{aligned}
$$



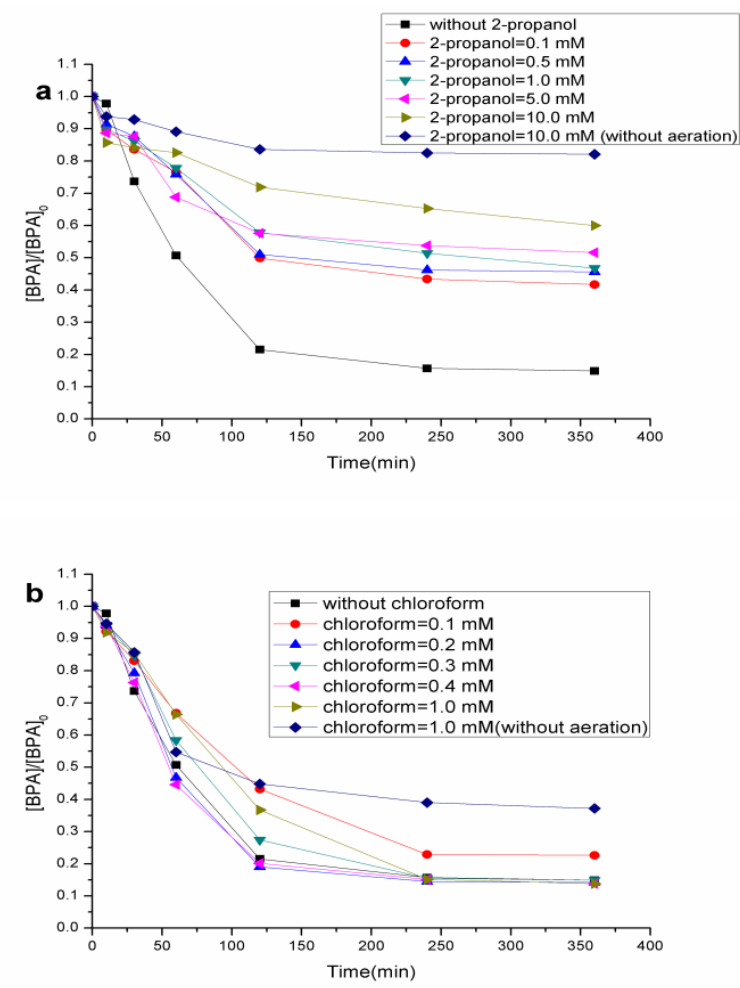

Fig. 5 BPA degradation in the modified photo-Fenton system in the presence of different scavenger $\mathbf{a}$ 2-propanol and $\mathbf{b}$ chloroform concentrations (conditions: $[E D D S]=1.0 \mathrm{mmol} \mathrm{L}^{-1}$, [ferrihydrite] $=0.6 \mathrm{~g} \mathrm{~L}^{-1},\left[\mathrm{H}_{2} \mathrm{O}_{2}\right]=0.5 \mathrm{mmol} \mathrm{L}^{-1},[\mathrm{BPA}]_{0}=20 \mu \mathrm{mol} \mathrm{L}$ 1 , $\mathrm{pH}=7)$.

Effects of iron dissolution. To further study the mechanism of this novel system, the iron dissolution in different systems was determined. The results of iron dissolution shown in Fig.6 are consistent with the experimental results of BPA degradation in different systems. In the presence of irradiation and EDDS, the iron dissolution and BPA degradation in the system were accelerated, indicating the role of iron dissolution in promoting the system efficiency. Some researchers have investigated the causes of increased iron dissolution in the presence of organic acids. ${ }^{60-62}$ They found that the surface complexes formed by organic acids and iron oxides could weaken the metal-oxygen bonds, thus increasing the amount of iron dissolution. Furthermore, the iron dissolution in the system enhanced the homogeneous Fenton reaction, through redox reaction of $\mathrm{H}_{2} \mathrm{O}_{2}$ with dissolved iron that further promotes the degradation of BPA. Comparison with the national standard (GB3838-2002) ${ }^{63}$ shows that the iron dissolution concentration produced in this system is far lower than the standard limit $\left(0.3 \mathrm{mg} \mathrm{L}^{-1}\right)$, therefore secondary pollution to the environment is not a concern.
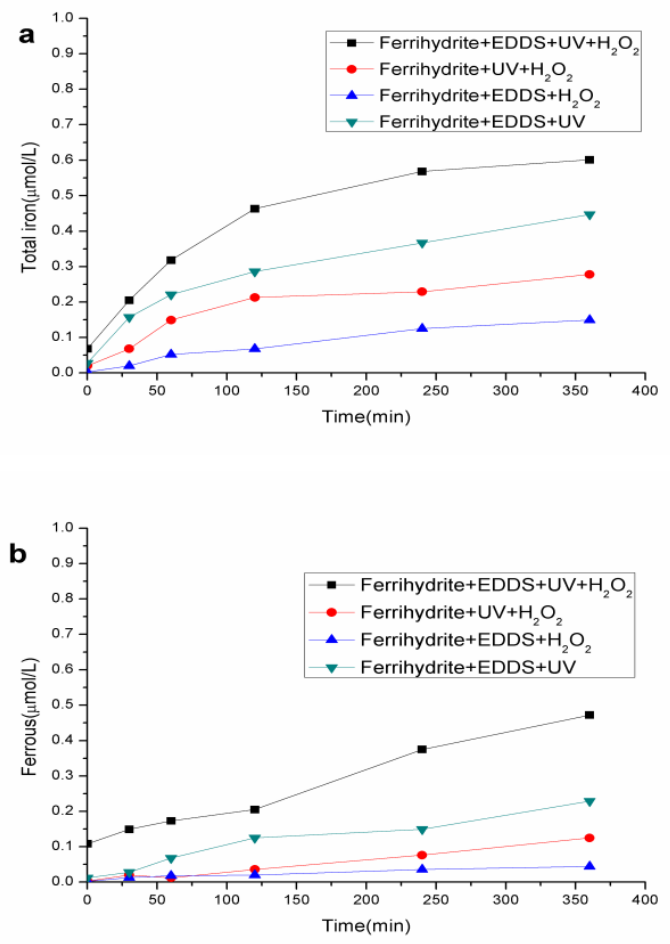

Fig. 6 a total iron dissolution rate dissolution and $\mathbf{b}$ ferrous dissolution rate in different systems (conditions: [EDDS] $=1.0$ mmol $\mathrm{L}^{-1}$, [ferrihydrite] $=0.6 \mathrm{~g} \mathrm{~L} \mathrm{~L}^{-1}, \quad\left[\mathrm{H}_{2} \mathrm{O}_{2}\right]=0.5 \mathrm{mmol} \mathrm{L}^{-1}$, $[B P A]_{0}=20 \mu \mathrm{mol} \mathrm{L}^{-1}, \mathrm{pH}=7$ ).

Proposed reaction mechanisms involved in the ferrihydriteEDDS/ photo-Fenton system. Based on the above results, a proposed mechanism of BPA degradation in this novel photoFenton system was determined, as shown in Fig. 6. Fig. 6 shows that BPA degradation can be attributed to several reactive species, including $\cdot \mathrm{OH}$ radicals, singlet oxygen and $\cdot \mathrm{O}_{2}^{-}$radicals. The first step of the reaction involves the formation of a complex between EDDS and ferrihydrite through charge transfer between the ligand and the metal. ${ }^{64}$ Then, electrons $\left(\mathrm{e}^{-}\right)$in the valence band (VB) of this $\mathrm{Fe}(\mathrm{III})$ EDDS complex under irradiation are excited into jump to the conduction band (CB), leaving holes $\left(\mathrm{h}^{+}\right)$in the valence band and forming $\mathrm{e}^{-}-\mathrm{h}^{+}$pairs (see Eq. (17)). ${ }^{59,65}$ Differences are observed in the mechanism of BPA degradation under aerobic and anaerobic conditions. On the one hand, in the aerobic condition, the presence of oxygen and holes at the ferrihydrite surface promotes the formation of ${ }^{1} \mathrm{O}_{2}$ which can directly oxidize BPA (see Eqs. (18)-(19)). Simultaneously, a very low yield of $\cdot \mathrm{O}_{2}{ }^{-}$radicals are produced due to the consumption of holes (see Eq. (20)). Furthermore, the foremost arrangement of the whole process was the reaction of $\mathrm{Fe}(\mathrm{II})$ with $\mathrm{H}_{2} \mathrm{O}_{2}$ to produce many $\cdot \mathrm{OH}$ (see Eq. (5)). As such, $\cdot \mathrm{OH}$ radicals and ${ }^{1} \mathrm{O}_{2}$ play important roles in aerobic conditions, while the role of $\cdot \mathrm{O}_{2}{ }^{-}$radicals is negligible. On the other hand, the formation of ${ }^{1} \mathrm{O}_{2}$ was absent under anaerobic conditions, meaning enough holes are available to oxidize $\mathrm{H}_{2} \mathrm{O}_{2}$ to $\cdot \mathrm{O}_{2}^{-}$radicals. The presence of $\cdot \mathrm{O}_{2}^{-}$radicals accelerated the reduction of $\mathrm{Fe}$ (III) to $\mathrm{Fe}(\mathrm{II})$, which contributed to the generation of $\cdot \mathrm{OH}$ radicals. 
Therefore, $\cdot \mathrm{O}_{2}^{-}$radicals are non-negligible under anaerobic conditions. In addition, the reaction between trace iron dissolution and $\mathrm{H}_{2} \mathrm{O}_{2}$ also promoted the $\mathrm{Fe}$ (II) formation. With the presence of $\cdot \mathrm{OH}$ radicals, ${ }^{1} \mathrm{O}_{2}$ and $\cdot \mathrm{O}_{2}^{-}$radicals, BPA could be degraded effectively in this novel photo-Fenton system.

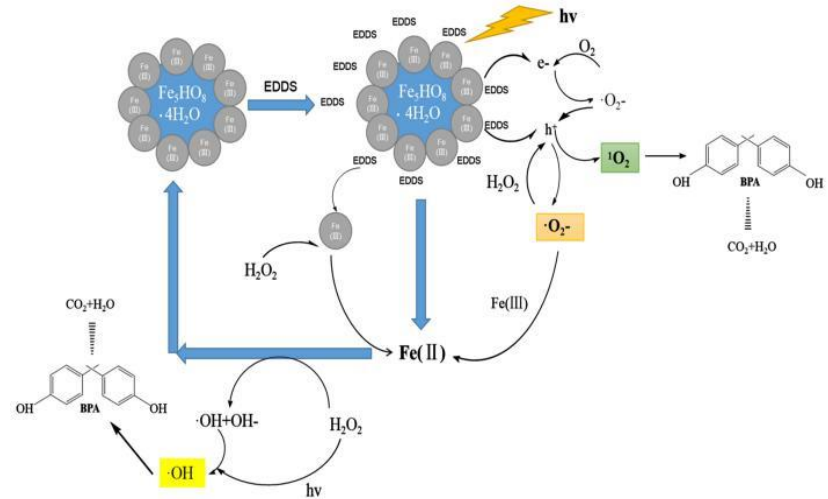

Fig. 7 Photodegradation mechanism of BPA in the ferrihydriteEDDS/photo-Fenton system

\section{Conclusions}

In this paper, the effect of the ferrihydrite-EDDS/photo-Fenton system on BPA degradation was investigated. We concluded that the introduction of irradiation and EDDS to the heterogeneous photo-Fenton system can effectively enhance the degradation of BPA at neutral $\mathrm{pH}$. Approximately $85.1 \%$ degradation of BPA was obtained after 360min under optimal experimental conditions ( $[\mathrm{EDDS}]=1.0 \quad \mathrm{mmol} \mathrm{L}^{-1}$ [ferrihydrite $]=0.6 \mathrm{~g} \mathrm{~L}^{-1},\left[\mathrm{H}_{2} \mathrm{O}_{2}\right]=0.5 \mathrm{mmol} \mathrm{L}{ }^{-1}$, $[\mathrm{BPA}]_{0}=20 \mu \mathrm{mol}$ $\mathrm{L}^{-1}, \mathrm{pH}=7$ ). In addition, the contribution of different reactive species, including $\cdot \mathrm{OH}$ radicals, $\cdot \mathrm{O}_{2}^{-}$radicals and ${ }^{1} \mathrm{O}_{2}$ were observed using radical scavengers. The generation of reactive species was responsible for BPA degradation under different conditions, with $\cdot \mathrm{OH}$ and ${ }^{1} \mathrm{O}_{2}$ appearing to have a primary effect in the aerobic condition while $\cdot \mathrm{O}_{2}^{-}$radicals demonstrated a non-negligible effect under anaerobic conditions. The results of the iron dissolution experiments showed that the iron dissolution was accelerated in the presence of irradiation and EDDS which promoted the system efficiency. The main aim of this paper is to improve the photo-Fenton system so that it can be better applied to sewage treatment. The EDDS is environmentally friendly, ferrihydrite is common and iron dissolves little.

\section{Acknowledgements}

This work was partially supported by National Natural Science Foundation of China (No. 21367003), Guangxi Science and Technology Research Program (No. AA17202032) and Open Fond of Guangxi Key Laboratory of Clean Pulp \& Papermaking and Pollution Control (KF201724).

\section{Notes and references}

1 Y. Feng, D. Wu, L. Ma. Iron oxide catalyzed Fenton-like reaction[J]. Progress in Chemistry, 2013.

2 Yang Xiaoling,Chen Wei,Huang Jianfei,Zhou Ying,Zhu Yihua,Li Chunzhong. Rapid degradation of methylene blue in a novel heterogeneous Fe3O4 @ rGO@TiO2-catalyzed photoFenton system.[J]. Scientific reports,2015,5

3 Xiaogang Zheng,Wendi Fu,Fuyan Kang,Hao Peng,Jing Wen. Enhanced photo-Fenton degradation of tetracycline using TiO 2 -coated $\alpha$-Fe 2 O 3 core-shell heterojunction[J]. Journal of Industrial and Engineering Chemistry, 2018.

4 Junge Xu,Yunqin Li,Baoling Yuan,Chunhua Shen,Minglai $\mathrm{Fu}$, Haojie Cui,Wenjie Sun. Large scale preparation of $\mathrm{Cu}$ doped $\alpha-\mathrm{FeOOH}$ nanoflowers and their photo-Fenton-like catalytic degradation of diclofenac sodium[J]. Chemical Engineering Journal,2016,291.

$5 \mathrm{Xu}$ Zhihui,Zhang Ming,Wu Jingyu,Liang Jianru,Zhou Lixiang,L Bo. Visible light-degradation of azo dye methyl orange using $\mathrm{TiO} 2 / \beta-\mathrm{FeOOH}$ as a heterogeneous photoFenton-like catalyst.[J]. Water science and technology : a journal of the International Association on Water Pollution Research,2013,68(10).

6 Wang X, Liu C, Li X, et al. Photodegradation of 2mercaptobenzothiazole in the $\gamma$-Fe2O3/oxalate suspension under UVA light irradiation[J]. Journal of Hazardous Materials, 2008, 153(1-2):426-433.

7 Shunitz Tanaka,Masayuki Kawai,Yosuke Nakata,Motoki Terashima,Hideki Kuramitz,Masami Fukushima. Degradation of bisphenol A by photo-fenton processes[J]. Toxicological \&amp; Environmental Chemistry,2003,85(4-6).

8 Walling C , Amarnath K . Oxidation of mandelic acid by Fenton $\backslash$ "s reagent[J]. Journal of the American Chemical Society, 1982, 104(5):1185-1189.

9 Tianyuan Xu,Runliang Zhu,Gangqiang Zhu,Jianxi Zhu,Xiaoliang Liang, Yanping Zhu,Hongping He. Mechanisms for the enhanced photo-Fenton activity of ferrihydrite modified with $\mathrm{BiVO} 4$ at neutral $\mathrm{pH}[\mathrm{J}]$. Applied Catalysis B: Environmental,2017,212.

$10 \mathrm{Zhu}$, Yanping, Zhu, Runliang, Xi, Yunfei,et al. Heterogeneous photo-Fenton degradation of bisphenol A over $\mathrm{Ag} / \mathrm{AgCl} /$ ferrihydrite catalysts under visible light[J]. Chemical Engineering Journal:S1385894718306466.

11 Yanping Zhu,Runliang Zhu,Lixia Yan,Haoyang Fu,Yunfei Xi,Huijun Zhou,Gangqiang Zhu,Jianxi Zhu,Hongping He. Visible-light $\mathrm{Ag} / \mathrm{AgBr} /$ ferrihydrite catalyst with enhanced heterogeneous photo-Fenton reactivity via electron transfer from $\mathrm{Ag} / \mathrm{AgBr}$ to ferrihydrite[J]. Applied Catalysis B: Environmental,2018.

12 Wang Tiecheng,Cao Yang,Qu Guangzhou,Sun Qiuhong,Xia Tianjiao,Guo Xuetao,Jia Hanzhong,Zhu Lingyan. Novel $\mathrm{Cu}(\mathrm{II})$-EDTA Decomplexation by Discharge Plasma Oxidation and Coupled $\mathrm{Cu}$ Removal by Alkaline Precipitation: Underneath Mechanisms.[J]. Environmental science \&amp; technology,2018,52(14).

13 Yongchun Dong,Peng Wang,Lu Gan,Bing Li,Hongjie Wen. Enhanced removal of aqueous $\mathrm{Cr}(\mathrm{VI})$ using optimized $\mathrm{Fe}$ complex on EDTA modified cotton fiber via photocatalytic reduction and adsorption hybrid functions[J]. Cellulose,2019,26(12).

14 Jin Wu,Jian Zhou,Shouwei Zhang,Ahmed Alsaedi,Tasawar Hayat,Jiaxing Li,Yuntao Song. Efficient removal of metal contaminants by EDTA modified MOF from aqueous solutions $[\mathrm{J}]$. Journal of Colloid And Interface Science, 2019,555.

15 Saraswati Saini,Jatinder Kaur Katnoria,Inderpreet Kaur. A comparative study for removal of cadmium(II) ions using unmodified and NTA-modified Dendrocalamus strictus 
charcoal powder[J]. Journal of Environmental Health Science and Engineering,2019,17(1).

16 Antonella De Luca,Renato F. Dantas,Santiago Esplugas. Study of Fe(III)-NTA chelates stability for applicability in photo-Fenton at neutral $\mathrm{pH}[\mathrm{J}]$. Applied Catalysis B Environmental,2015,179.

17 Wenyu Huang,Mengqi Luo,Chaoshuai Wei, Yinghui Wang,Khalil Hanna,Gilles Mailhot. Enhanced heterogeneous photo-Fenton process modified by magnetite and EDDS BPA degradation[J]. Environmental Science and Pollution Research,2017,24(11).

18 Jing Li,Gilles Mailhot,Feng Wu,Nansheng Deng. Photochemical efficiency of Fe(III)-EDDS complex: $<$ ce:glyph name $="$ rad" $>\mathrm{OH}$ radical production and $17 \beta$-estradiol degradation[J]. Journal of Photochemistry \&amp; Photobiology, A: Chemistry,2010,212(1).</ce:glyph>

19 Patisaul Heather B. Achieving CLARITY on bisphenol A, brain and behaviour.[J]. Journal of neuroendocrinology,2019.

20 F S vom Saal. Triennial Reproduction Symposium: Environmental programming of reproduction during fetal life: Effects of intrauterine position and the endocrine disrupting chemical bisphenol A $1[\mathrm{~J}]$. Journal of Animal Science, 2016,94(7).

21 Shengman Wang,Lihong Wang,Weiqi Hua,Min Zhou,Qingqing Wang,Qing Zhou,Xiaohua Huang. Effects of bisphenol A, an environmental endocrine disruptor, on the endogenous hormones of plants[J]. Environmental Science and Pollution Research,2015,22(22).

22 U. Schwertmann, R. M. Cornell. Iron Oxides in Laboratory[J]. Soil Science, 1993, 156(5):281-282.

23 Sangchul Hwang,Scott G. Huling,Saebom Ko. Fenton-like degradation of MTBE: Effects of iron counter anion and radical scavengers[J]. Chemosphere,2009,78(5).

24 Buxton G V, Greenstock C L, Helman W P, et al. Critical Review of rate constants for reactions of hydrated electrons, hydrogen atoms and hydroxyl radicals $\left(\cdot \mathrm{OH} / \cdot \mathrm{O}^{-}\right.$in Aqueous Solution[J]. Journal of Physical and Chemical Reference Data, 2009, 17(2):513-886

25 Tamura H,Goto K,Yotsuyanagi T,Nagayama M Spectrophotometric determination of iron(II) with 1,10phenanthroline in the presence of large amounts of iron(III). [J]. Talanta, 1974,21(4).

26 J.L. Jambor, J.E. Dutrizac, Occurrence and constitution of natural and synthetic ferrihydrite, a widespread iron oxyhydroxide, Chem. Rev. 98 (1998) 2549-2585.

27 Ma Y, Meng S, Qin M, et al. New insight on kinetics of catalytic decomposition of hydrogen peroxide on ferrihydrite: Based on the preparation procedures of ferrihydrite $[\mathrm{J}]$. Journal of Physics and Chemistry of Solids, 2012, 73(1): 3034.

28 Carta D , Casula M F , Corrias A, et al. Structural and magnetic characterization of synthetic ferrihydrite nanoparticles[J]. Materials Chemistry and Physics, 2009, 113(1):349-355.

29 Gautier J , Grosbois C , Courtin- Nomade A. Transformation of natural As - associated ferrihydrite downstream of a remediated mining site $[\mathbf{J}]$. European Journal of Mineralogy, 2006, 18 (2) : 187-195.

30 Jia Y, Xu L, Wang X, et al. Infrared spectroscopic and $\mathrm{X}$-ray diffraction characterization of the nature of adsorbed arsenate on ferrihydrite $[\mathrm{J}]$. Geochimica $\mathrm{Et}$ Cosmochimica Acta, 2007, 71( 7) : 1643

31 Rout K, Mohapatra M, Anand S . 2-Line ferrihydrite: synthesis, characterization and its adsorption behaviour for removal of $\mathrm{Pb}(\mathrm{II}), \mathrm{Cd}(\mathrm{II}), \mathrm{Cu}(\mathrm{II})$ and $\mathrm{Zn}(\mathrm{II})$ from aqueous solutions[J]. Dalton Transactions, 2012, 41.

32 Garau Giovanni,Lauro Gian Paolo,Diquattro Stefania,Garau Matteo,Castaldi Paola. Sb(V) adsorption and desorption onto ferrihydrite: influence of $\mathrm{pH}$ and competing organic and inorganic anions.[J]. Environmental science and pollution research international,2019,26(26)

33 Liao P, Li W, Wang D, et al. Effect of reduced humic acid on the transport of ferrihydrite nanoparticles under anoxic conditions[J]. Water research, 2017, 109: 347-357.

34 Chenju Liang,Ching-Ping Liang,Chi-Chin Chen. pH dependence of persulfate activation by EDTA/Fe(III) for degradation of trichloroethylene[J]. Journal of Contaminant Hydrology,2009, 106(3).

35 Abida O, Mailhot G, Litter M, et al. Impact of iron-complex (Fe(III)-NTA) on photoinduced degradation of 4chlorophenol in aqueous solution[J]. Photochemical \& Photobiological Sciences, 2006, 5(4):395.

$36 \mathrm{Wu}$ Yanlin,Brigante Marcello,Dong Wenbo,de Sainte-Claire Pascal,Mailhot Gilles. Toward a better understanding of Fe(III)-EDDS photochemistry: theoretical stability calculation and experimental investigation of 4-tertbutylphenol degradation.[J]. The journal of physical chemistry. A,2014,118(2).

37 Huang Wenyu,Brigante Marcello,Wu Feng,Hanna Khalil,Mailhot Gilles. Effect of ethylenediamine-N,N'disuccinic acid on Fenton and photo-Fenton processes using goethite as an iron source: optimization of parameters for bisphenol A degradation.[J]. Environmental Science and Pollution Research,2013.

38 Vandevivere P C,Saveyn H,Verstraete W,Feijtel T C,Schowanek D R. Biodegradation of metal-[S,S]-EDDS complexes.[J]. Environmental Science \&amp; Technology,2001,35(9).

39 Kaiqun Wu,Yinde Xie,Jincai Zhao,Hisao Hidaka. PhotoFenton degradation of a dye under visible light irradiation $[\mathrm{J}]$. Journal of Molecular Catalysis. A, Chemical,1999,144(1).

40 E. Neyens,J. Baeyens. A review of classic Fenton's peroxidation as an advanced oxidation technique $[\mathrm{J}]$. Journal of Hazardous Materials,2003,98(1).

41 Ruixiong Huang,Zhanqiang Fang,Xiaomin Yan,Wen Cheng. Heterogeneous sono-Fenton catalytic degradation of bisphenol A by $\mathrm{Fe} 3 \mathrm{O} 4$ magnetic nanoparticles under neutral condition[J]. Chemical Engineering Journal,2012,197.

42 Christopher M Miller, Richard L Valentine. Mechanistic studies of surface catalyzed $\mathrm{H} 2 \mathrm{O} 2$ decomposition and contaminant degradation in the presence of sand[J]. Water Research, 33(12):0-2816.

43 Schrank S G, José H J, Moreira R F P M, et al. Applicability of Fenton and $\mathrm{H} 2 \mathrm{O} 2 / \mathrm{UV}$ reactions in the treatment of tannery wastewaters[J]. Chemosphere, 2005, 60(5):644-655.

44 Jiangkun Du,Jianguo Bao,Xiaoyan Fu,Chenghang Lu,Sang Hoon Kim. Mesoporous sulfur-modified iron oxide as an effective Fenton-like catalyst for degradation of bisphenol A[J]. Applied Catalysis B: Environmental,2016,184.

$45 \mathrm{Li} \mathrm{W}$, Wang $\mathrm{Y}$, Irini A. Effect of $\mathrm{pH}$ and $\mathrm{H} 2 \mathrm{O} 2$ dosage on catechol oxidation in nano-Fe3O4 catalyzing UV-Fenton and identification of reactive oxygen species[J]. Chemical Engineering Journal, 2014, 244:1-8.

46 Avetta P , Pensato A , Minella M , et al. Activation of Persulfate by Irradiated Magnetite: Implications for the Degradation of Phenol under Heterogeneous Photo-FentonLike Conditions[J]. Environmental Science and Technology, 2014, 49(2):1043.

47 Li J, Mailhot G, Wu F, et al. Photodegradation of E2 in the presence of natural Montmorillonite and the iron complexing agent ethylenediamine-N,N'-disuccinic acid[J]. Photochemical and Photobiological Sciences, 2012, 11(12).

48 R.M. Cornell, U. Schwertmann, The iron oxides: structure, properties,reactions, occurrences and uses, John Wiley \& Sons, New York, 2006. 
49 Ding Yaobin,Xia Xiangli,Ruan Yufeng,Tang Heqing. In situ $\mathrm{H}(+)$-mediated formation of singlet oxygen from $\mathrm{NaBiO} 3$ for oxidative degradation of bisphenol A without light irradiation: Efficiency, kinetics, and mechanism.[J]. Chemosphere, 2015,141

50 Doorslaer X V, Heynderickx P M, Demeestere K, et al. TiO 2 mediated heterogeneous photocatalytic degradation of moxifloxacin: Operational variables and scavenger study[J]. Applied Catalysis B Environmental, 2012, 111(112):150-156.

51 Palominos R, Freer J, Mondaca M A, et al. Evidence for hole participation during the photocatalytic oxidation of the antibiotic flumequine[J]. Journal of Photochemistry \& Photobiology A Chemistry, 2008, 193(2-3):139-145.

52 Zhang X, Darren D, Guoting L I, et al. Investigation of the roles of active oxygen species in photodegradation of azo dye $\mathrm{A} 07$ in $\mathrm{TiO} 2$ photocatalysis illuminated by microwave electrodeless lamp[J]. Journal of Photochemistry \& Photobiology A Chemistry, 2008, 199(2-3):311-315.

53 Chun Zhao,Miguel Pelaez,Xiaodi Duan, et al. Role of pH on photolytic and photocatalytic degradation of antibiotic oxytetracycline in aqueous solution under visible/solar light: Kinetics and mechanism studies[J]. Applied Catalysis B: Environmental, 2013,134-135.

54 Julie R. Peller, Stephen P. Mezyk, William J. Cooper. Bisphenol A reactions with hydroxyl radicals: diverse pathways determined between deionized water and tertiary treated wastewater solutions[J]. 35(1):21-34

55 Chen X, Ma W, Li J , et al. Photocatalytic Oxidation of Organic Pollutants Catalyzed by an Iron Complex at Biocompatible $\mathrm{pH}$ Values: Using $\mathrm{O} \backslash \mathrm{r}, 2 \backslash \mathrm{r}$, as Main Oxidant in a Fenton-like Reaction[J]. The Journal of Physical Chemistry C, 2011, 115(10):4089-4095.

56 Torres R A, Pétrier, Christian, Combet E, et al. Bisphenol A Mineralization by Integrated Ultrasound-UV-Iron (II) Treatment[J]. Environmental Science \& Technology, 2007, 41(1):297-302.

57 Hwang S , Huling S G , Ko S . Fenton-like degradation of MTBE: Effects of iron counter anion and radical scavengers[J]. Chemosphere, 2010, 78(5):0-568.

$58 \mathrm{He}$ Weiwei,Jia Huimin,Yang Dongfang,Xiao Pin,Fan Xiaoli,Zheng Zhi,Kim Hyun-Kyung,Wamer Wayne G,Yin Jun-Jie. Composition Directed Generation of Reactive Oxygen Species in Irradiated Mixed Metal Sulfides Correlated with Their Photocatalytic Activities.[J]. ACS applied materials \&amp; interfaces,2015,7(30).

59 Nosaka, Yoshio, Nosaka, Atsuko Y. Generation and Detection of Reactive Oxygen Species in Photocatalysis[J]. Chemical Reviews:acs.chemrev.7b00161.

60 Sposito G. Chemistry of the solid-water interface : W. Stumm (with contributions by L. Sigg and B. Sulzberger). John Wiley \& Sons, Chichester, 1992, paperback, $\mathrm{x}+428$ pp. price $£ 32.50$. ISBN 0-471-57672-7[J]. 1993, 42(3-4):259-260.

61 Stumm W. Reactivity at the mineral-water interface: dissolution and inhibition[J]. 1997, 120(1-3):143-166.

62 W.P. Miller, L.W. Zelazny, D.C. Martens. Dissolution of synthetic crystalline and noncrystalline iron oxides by organic acids[J]. Geoderma, 37(1):0-13.

63 S. Yu, G. B. Yu, Y. Liu,. Urbanization Impairs Surface Water Quality: Eutrophication and Metal Stress In The Grand Canal Of China[J]. River Research \& Applications, 2012, 28(8).

64 Abida Otman,Kolar Michal,Jirkovsky Jaromir,Mailhot Gilles. Degradation of 4-chlorophenol in aqueous solution photoinduced by $\mathrm{Fe}(\mathrm{iii})$-citrate complex.[J]. Photochemical \&amp; Photobiological Sciences, 2012.

65 Ju L, Dai Y, Wei W, et al. Potential of one-dimensional blue phosphorene nanotubes as a water splitting photocatalyst[J]. Journal of Materials Chemistry A, 2018, 6. 